\title{
The State of Supply Chain Carbon Footprinting: Analysis of CDP Disclosures by US Firms
}

Christian Blanco ${ }^{\mathrm{a}, \mathrm{b}}$, Felipe Caro ${ }^{\mathrm{a}}$, Charles J. Corbett ${ }^{\mathrm{a}}$

${ }^{\mathrm{a}}$ UCLA Anderson School of Management

110 Westwood Plaza, Box 951481

Los Angeles, CA 90095, USA

cblanco@anderson.ucla.edu

felipe.caro@anderson.ucla.edu

charles.corbett@anderson.ucla.edu

${ }^{\mathrm{b}}$ corresponding author

\begin{abstract}
Although collaboration and performance measurement are widely recognized as critical for increasing sustainability in supply chains, little is known about how comprehensively firms are currently measuring their supply chain carbon emissions (or Scope 3). We develop a way to assess how complete firms' reports of Scope 3 emissions are, by comparing them to benchmarks based onEnvironmental Input-Output Life-Cycle Assessment models. We use this approach to evaluate Scope 3 carbon emissions disclosed by many of the largest firms in the United States to CDP (formerly the Carbon Disclosure Project). We estimate that, on average, these firms reported 22\% of their full Scope 3 emissions in 2013. Our results show that Scope 3 reporting varies widely across industries. We highlight two firms that are able to capture a significant portion of their Scope 3 carbon emissions despite having a large number of suppliers. Although
\end{abstract}


firms are beginning to account for an increasing portion of their supply chain's carbon emissions, there is substantial room for improvement in their measurement and disclosure of Scope 3 emissions.

Keywords: sustainable supply chains, carbon footprint, Scope 3 emissions, carbon disclosure.

\section{Introduction}

It is well-known that, for a supply chain to be more sustainable, firms in that supply chain need to collaborate more closely. From their extensive literature review, Seuring and Müller (2008, pp. 1705-6) conclude that "There is a much increased need for cooperation among partnering companies in sustainable supply chain management", with the reminder that this should involve looking "at a longer part of the supply chain". Performance measurement is naturally a critical but difficult part of that, as Seuring and Gold (2013) note in their introduction to a special issue of the Journal of Cleaner Production. Several recent papers confirm the importance of a supply chain perspective. Looking at the retail sector, Tidy et al. (2015, p. 16) report that Tesco estimates that $85 \%$ of its products' carbon footprint lies upstream, while Asda estimates that to be $90 \%$. Focusing on the energy sector, Dragomir (2012, p. 228) observes that BP estimates its Scope $3^{1}$ emissions to be 15 times greater than those from company operations. Ozawa-Meida et al. (2013) measure the carbon footprint of a university, and find that $79 \%$ of greenhouse gas emissions fall within Scope 3. This is all highly consistent with Matthews et al.

\footnotetext{
${ }^{1}$ Recall that a firm's greenhouse gas emissions are classified, by the Greenhouse Gas Protocol, as being in Scope 1 (from direct company equipment), Scope 2 (due to electricity purchases), or Scope 3 (indirect due to activities elsewhere in their supply chain).
} 
(2008), henceforth MHW08, who estimate that, averaging across all US sectors, upstream Scope 3 emissions represent $74 \%$ of a firm's total footprint.

If Scope 3 emissions are such a large part of a firm's supply-chain carbon footprint, it becomes important to understand the extent to which firms are measuring those Scope 3 emissions. Boström et al. (2015, p. 3) highlight that one of the major challenges in improving sustainable supply chain governance is information gaps about the sustainability impacts of products and processes throughout a supply chain. There is currently no obligation for firms to report Scope 3 emissions at all, though Tidy et al. (2015) report that some companies anticipate that such a legal requirement may be on the way. The importance of Scope 3 is also highlighted by the development of several reporting standardssuch as the Global Reporting Initiative (GRI) G4 Sustainability Reporting Guidelinesand the Greenhouse Gas (GHG) Protocol.

Many firms do voluntarily disclose Scope 3 emissions in their sustainability reports, often following the GRI G4 Sustainability Reporting Guidelines, or to organizations like CDP (formerly the Carbon Disclosure Project), a UK-based organization that holds the largest global collection of firm-reported climate-change-related data. ${ }^{2}$ The GRI and the CDP has also recently aligned reporting standards to ease the reporting burden on companies and allow them to use the same data in both frameworks (Basacik et al. 2015). For example, The GRI G4 Sustainability Reporting Guidelines was updated for the fourth time (thus the term G4) in May 2013 to align some environmental indicators with other reporting standards such as CDP. For example, the GRI indicator G4-EN17 is now compatible with Scope 3 as defined by the GHG Protocol (GRI 2015, p. 58). The changes to the G4 reporting guidance reflect the increasing shift in attention to

\footnotetext{
${ }^{2}$ See https://www.cdp.net/en-US/Pages/About-Us.aspx, last accessed October 28, 2015.
} 
supply chain carbon emissions (GRI 2013) and the alignment of Scope 3 reporting to CDP in the GRI framework (Basacik et al. 2015, p.5).

Disclosures to CDP of Scope 1 and 2 emissions are expected to be complete (but are not necessarily verified); Scope 3 disclosures are entirely discretionary. Even among firms that do report to CDP, many do not report Scope 3 at all: in Dragomir's (2012) sample, only two of the five energy firms report any Scope 3 emissions (Table 7, p. 234). Even when firms do report, it is unclear how to interpret the disclosures. Although 265 US firms did report some Scope 3 emissions to CDP in 2013 (out of 397 firms who disclosed at all), CDP itself notes (CDP 2014b) that "current Scope 3 reporting does not reflect the full impact of companies' activities, and may mislead as to the full carbon impact of a company". In one of the very few studies focusing explicitly on how firms report Scope 3 emissions, Downie and Stubbs (2013) find that in their survey of 22 Australian companies, there is a lack of rigor on what sources of emissions to include. Talbot and Boiral (2013, p. 1077) point to the lack of guidance as a particular concern for Scope 3 emissions. Huang et al. (2009), henceforth HWM09, point out that the breakdown of emissions, and the main sources of Scope 3 emissions, vary by industry group, arguing that such guidance should hence be industry-specific.

CDP provides a comprehensive and easily accessible database of firm-reported Scopes 1, 2, and 3 , but because of the secondary nature of this data, it is difficult to verify its completeness. It is clear that Scope 3 reporting, if it happens at all, will be incomplete.However, what is currently lacking is a systematic assessment of how incomplete current Scope 3 reporting to CDP is. That is the contribution of this paper. Specifically, we examine the CDP disclosures by US firms, and contrast the resulting breakdown of emissions into Scope 1 and 2 vs. Scope 3 with the breakdown implied in MHW08. Using MHW08 as a benchmark, we estimate that US firms that 
reported any Scope 3 emissions to CDP in 2013 only captured 22\% of their total Scope 3 emissions. We then assess how that breakdown has evolved over time, and find that firms are capturing a greater portion of their Scope 3 emissions. We repeat our analysis for each industry separately, and find that some sectors have much more comprehensive Scope 3 disclosures than others. Finally, we briefly discuss how two companies were able to increase their Scope 3 reports from (almost) zero to becoming quite comprehensive within just a few years.

\section{Literature Review}

The growth in measuring and disclosing greenhouse gas emissions has led to a corresponding growth in related literature. The review byStechemesser and Guenther (2012) identified 129 papers on carbon accounting, covering the national, project, organizational and product scale. To extend their review past 2012, we identified academic papers that have estimated Scope 3 using the archival research method suggested by Searcy and Mentzer (2013) and as demonstrated by Sanches-Pereira et al. (2016) in their literature review. We focus on more recent papers published after October 2012, covering the periods after the analysis of Stechemesser and Guenther (2012). Our database source is the Web of Science, an online scientific citation indexing service (http://portal.isiknowledge.com/, last accessed February 18, 2016), and the unit of analysis is supply chain carbon emissions. We then narrowed the context to the following three key phrases: "Scope 3", "carbon footprinting", and "supply chains". We identified six published papers in English that are relevant to Scope 3 supply chain carbon footprintingfrom November 2012 to January 2016. Below we draw on literature cited by Stechemesser and Guenther (2012), the six more recent papers that we identified, and other related research. 
Some work has started to address Scope 3 emissions, though there is very little on the actual measurement of those emissions. Here we review literature on the benefits of measuring carbon emissions within the supply chain, which helps explain why firms disclose Scope 3 emissions at all given that it is voluntary. We then look at empirical studies that estimate supply chain carbon emissions followed by a discussion on the challenges of collecting and using Scope 3 information. We also provide some background on voluntary disclosure and the quality and usefulness of current voluntary carbon emission reporting.

Information about supplier vulnerability to climate change and greenhouse gas regulation enables companies to make better decisions and reduce risks associated with carbon emissions (Jira and Toffel 2013). Without an understanding of upstream emissions, firms may miss out on the most cost-effective carbon mitigation strategies (MHW08), especially given that such a large portion of emissions come from the supply chain.

Measuring carbon emissions within the supply chain has significant benefits, but adoption has been slow. Collaborating with suppliers to minimize pollution has a significant impact on both manufacturing and environmental performance (Vachon and Klassen 2008). Walmart was able to profitably reduce supply chain carbon emissions by engaging their suppliers to pursue such opportunities (Plambeck 2012). Suppliers are more likely to disclose carbon emissions if they face more requests from buyers and if buyers appear more committed to using it (Jira and Toffel 2013). Firms face increasing pressure to measure supply chain emissions (Jira and Toffel 2013; Reid and Toffel 2009), which helps explain why Scope 3 disclosure is increasing albeit still incomplete. 
We summarize the list of studies that have started to estimate Scope 3 in specific industries. Estimating the carbon emissions of Australian ambulance services, Brown et al. (2012) use a combination of ambulance data and Environmental Input-output life-cycle assessment (EIOLCA) models, and find that Scope 3 accounts for $58 \%$ of total carbon emissions in that industry. Looking at the largest research institute in Mexico, Güereca et al. (2013) find that the fraction of Scope 3 is $53 \%$ of their total carbon emissions. Estimating carbon emissions for the US cheese and whey industry, Kim et al. (2013) show the importance of establishing a benchmark for supply chain carbon emissions. Analyzing carbon emissions in US commercial buildings, Onatet al. (2014) show that commuting accounts for the largest portion of Scope 3. These studies show the increasing interest in measuring carbon emissions in specific industries.

There are studies that estimate the breakdown of economy-wide Scopes 1, 2, and 3 using the EIOLCA method. Using EIOLCA models, Yang and Chen (2014) estimate sector-specific Scope 3 emissions for the Chinese economy. They find that 22 out of 29 industries have less than $20 \%$ of their total carbon footprint represented by Scope 1 and 2. HWM09 is the first paper to estimate sector-specific Scope 3 emissions for the entire US economy. HWM09 use EIOLCA to identify sources of upstream emissions that contribute the most to each sector's footprints. They show that a large portion of Scope 3 emissions can be captured from a small number of supplier sectors. For example, for manufacturing sectors, $50 \%-70 \%$ of their total upstream Scope 3 footprint can be accounted for by the industry's top 10 supplier sectors. HWM09 suggest that Scope 3 capture rates can be improved considerably by providing industry-specific reporting protocols. Our contribution is to provide the link between firm-reported Scope 3 and industrylevel Scope 3 estimates in the US economy. 
Firms face many challenges in collecting carbon emissions beyond company boundaries. An earlier study, also using CDP data, finds that only a little over half of all suppliers that are requested to share climate change information respond (Jira and Toffel 2013). Even when carbon emissions data is available, it may still be challenging to assess the quality of the data (Kolk et al. 2008; Melville and Whisnant 2014). The use of carbon emissions as a performance metric has also led to many discussions on how far upstream should carbon emissions be measured and how to assess its completeness (Busch 2010; Busch 2011; Hoffmann and Busch 2008; Murray et al. 2011). Aside from the time and resources needed to collect data, firms have yet to understand which sources of emissions to include and how to calculate them (Huang et al. 2009b). The type and scope of carbon emissions data collected will largely be driven by what will influence stakeholders (Marland et al. 2013). However, without a benchmark, it is difficult to assess how comprehensive and successful firms are in collecting Scope 3 information.

Firms are not yet taking advantage of the easy availability of data of EIOLCA models and their ability to capture emissions regardless of how far up the supply chain. In a survey of 22 Australian companies, none use Input-Output models to measure their supply chain emissions (Downie and Stubbs 2013). Our study gives insights on the current completeness of supply chain carbon reporting using data from the largest firms in the US and comparing that to EIOLCA.

The literature cited above is predicated on the premise that the majority of emissions occur upstream. MHW08 and HWM09 provide the only quantitative estimates of that breakdown, using industry-level EIOLCA. Our contribution is to contrast the top-down estimate of the breakdown of emissions from EIOLCA with a bottom-up estimate based on firm-level disclosures of Scope 3 emissions. CDP has successfully leveraged institutional investors to encourage firms to disclose (Kolk et al. 2008), but despite this, the quality and 
comprehensiveness of those disclosures still shows room for improvement (Kolk et al.

2008; Matisoff et al. 2013; Sullivan and Gouldson 2012). We provide a different way to assess the completeness of Scope 3 disclosure and show how it has improved over time.

The reports on the CDP website provide detailed commentary on emissions trends, by country, by sector, and over time. One of the main findings in their 2013 report was that companies are sometimes able to identify the most carbon-intensive activities (or hot spots) from their supply chain, but emissions from these activities are yet to be quantified (CDP 2014b).

\section{Materials and Methods}

We use the CDP database to construct a firm-level counterpart to MHW08 and HWM09. HWM09 build on MHW08 to obtain Scope 3 estimates by industry. Following MHW08 and HWM09, we define Scope 3 as emissions from sources that are not owned or controlled by the company, but that occur within the company's upstream value chain, such as business travel, purchased goods and services, and other upstream activities. In some contexts, Scope 3 includes the company's downstream value chain, but, to be consistent with MHW08 and HWM09, we exclude those here. CDP follows Scope 3 reporting standards provided by the Greenhouse Gas Protocol, which are consistent and directly comparable with the Intergovernmental Panel on Climate Change (WRI/WBCSD, 2004).

CDP provides all historical survey responses to their climate-change information request survey. Researchers can access all historical CDP data through the CDP academic data package website (CDP 2016). Each year since 2003, CDP invites firms with the largest market capitalization to take their survey, on behalf of 722 investors with assets of US\$87 trillion as of 
2013 (CDP 2014a). The survey questions are also available on their website (CDP 2013). We use CDP disclosures of Scope 1, 2 and 3 emissions by US firms. For example, the responses to survey questions 9 and 10 in the Investor CDP 2013 Information Request survey (CDP 2013) were used for Scope 1 and 2. Survey question 9 allows firms to provide Scope 1 by country: "Scope 1 Emissions Breakdown: Do you have Scope 1 emissions sources in more than one country?" If the firm responds yes, then they are asked to provide Scope 1 for each country. Survey question 10 is the same as 9, but for Scope 2. We focus on Scope 1 and 2 emissions that occur in the US. For companies that do not have emissions outside the US, their global Scope 1 and 2 emissions are equivalent to their US emissions. Scope 3 is obtained from responses to survey question 14 from the CDP 2013 Information Request survey: "Scope 3 Emissions: Please account for your organization's Scope 3 emissions." The CDP surveys do not breakdown Scope 3 by country. This will make our Scope 3 larger compared to using Scope 3 only within the US. We discuss the implications of this in more detail in Section 4.1.

CDP includes the full list of S\&P 500 firms in their annual S\&P 500 report, and they highlight Scopes 1, 2, and 3 reported by the top performing firms in each sector. For example, the 2009 CDP S\&P 500 report shows that even some of the firms with the highest Carbon Disclosure Leadership Index ranking do not report Scope 3 (see for example CDP (2009) Figure E, p. 16). Moreover, even if firms do report emissions from all Scopes 1, 2, and 3, it is still difficult to determine whether firms are reporting a significant amount of their Scope 3 emissions.For example, the 2013 CDP S\&P 500 report (Appendix I) shows the ratio of the number of categories of Scope 3 emission sources that each firm has identified as "relevant, calculated" to the number of Scope 3 emissions categories identified as "relevant, calculated", "relevant, not calculated", "not evaluated", and no response to assess the completeness of their 
Scope 3 reporting (CDP 2013b). However, this does not take into account the amount of Scope 3 emissions actually reported relative to Scopes 1 and 2. For example, Boeing, an aircraft manufacturing company, scored a 7\% using the CDP Scope 3 progress metric, with only 1 out of 14 categories identified as "relevant, calculated". In contrast, Best Buy, an American multinational consumer electronics retailer, scored 3 out of 5 , meaning that 3 out of 5 categories of Scope 3 emissions are "relevant, calculated". This gives them a score of $60 \%$. A firm may report emissions from all categories, but it is not clear how this compares to Scopes 1 and 2. The approach we propose uses Scopes 1, 2, and 3.

Next, we describe how we establish a link between firm-reported data and the Environmental Input-Output Life-Cycle Assessment models. To compare the firm-level CDP disclosures to the industry-level breakdown of emissions in HWM09, we have to match the firms in the CDP dataset to the industries distinguished by HWM09. To do so, we use the primary North American Industry Classification System (NAICS) code listed in Compustat, which is a database of financial information on global companies. Compustat is widely used by institutional investors, academics, and analysts, and started including primary NAICS codes in 2000 . The CUSIP (Committee on Uniform Security Identification Procedures) $\operatorname{codes}^{3}$ in the CDP dataset are used to match each firm to the Compustat database to obtain each firm's NAICS code. Throughout, we determine the weighted average breakdown of emissions within each industry. For the economy-wide breakdown, we then use the arithmetic average across industries.

The number of firms voluntarily disclosing has steadily increased since 2003. In 2005, about 100 US firms disclosed, up to 334 firms in the S\&P 500 index in 2013 (CDP 2014a). Figure 1

\footnotetext{
${ }^{3}$ A CUSIP code is a nine character alphanumeric code to identify North American companies.
} 
shows that, in 2013, 397 US firms disclosed to CDP, of which 265 reported upstream Scope 3 emissions.

Figure 1. Number of US firms (including non-S\&P500) disclosing climate-change-related data and upstream Scope 3 emissions to CDP.

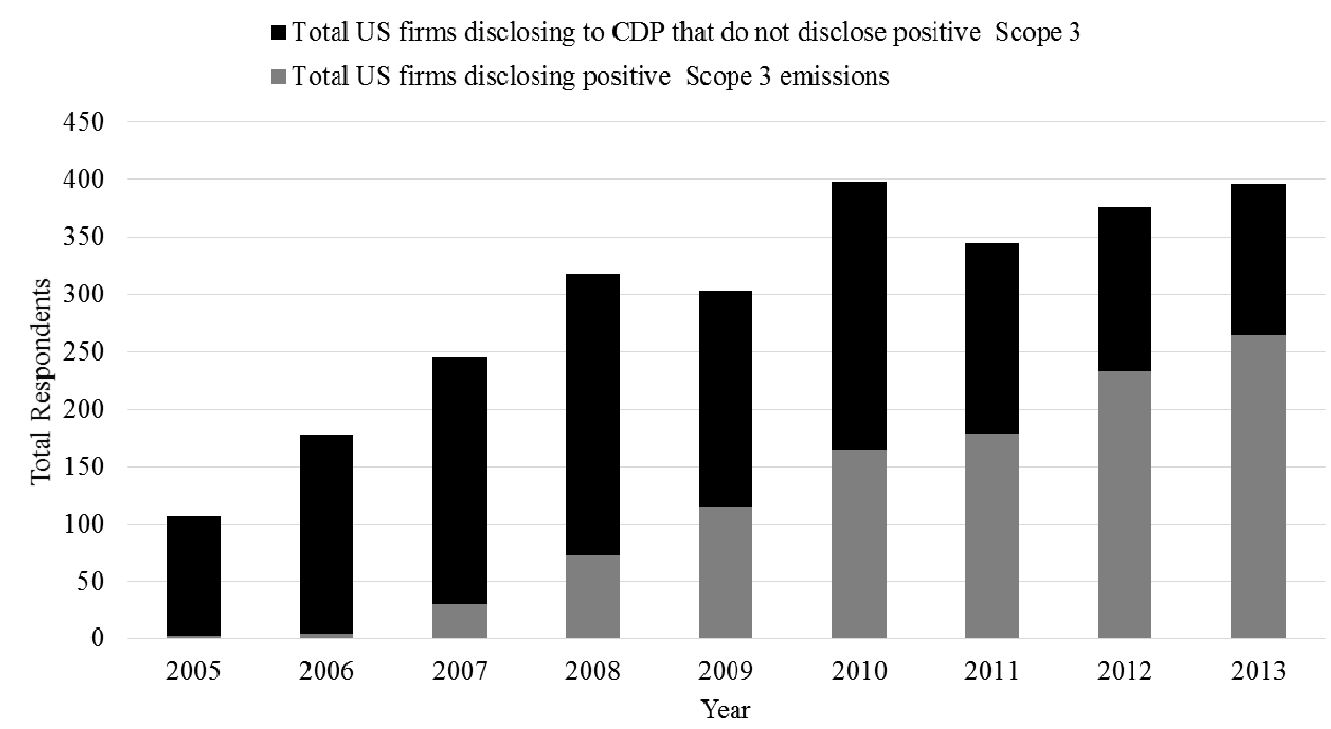

Figure 2 shows the increasing evolution of the actual emissions disclosed to CDP by US respondents. The top area shows total Scope 1 and 2 emissions from respondents who did not disclose any Scope 3 that year. The middle area is total Scope 1 and 2 emissions for those respondents who disclosed at least one category of Scope 3 in that year, and the bottom area is the total Scope 3 for those same respondents. Firms are not required to include emissions from all tiers of suppliers, but they are encouraged to collect the information from as many sources as they can identify, as outlined by the Greenhouse Gas Protocol (The Greenhouse Gas Protocol, 2015, p.34-37). Firms are disclosing more Scope 1 and 2 as well as more Scope 3 emissions over 
time. The focus of this study is to see what fraction of their actual Scope 3 emissions firms are reporting, so we limit our sample to firms that report at least some emissions from Scope 1 and 2 as well as from Scope 3, i.e., the bottom and middle areas of Figure 2. If a firm discloses no Scope 3 emissions, it is clear that they under-report those emissions by $100 \%$; it is for those firms that do disclose some Scope 3 emissions that we wish to estimate by how much they under-report. If we included all firms, the extent of under-reporting would be even greater than we describe here.

Figure 2. Emissions disclosed to CDP over time, US respondents.

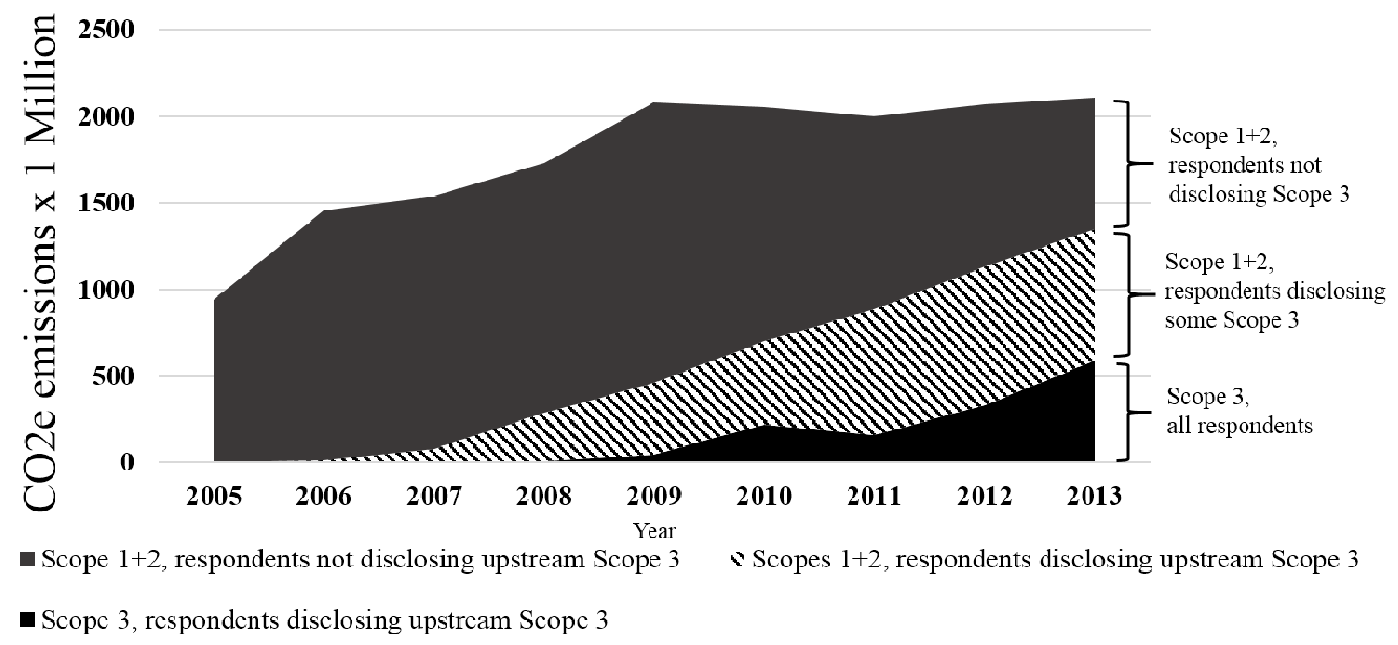

Although many firms do not disclose to CDP, it is nevertheless an informative sample, for two reasons. First, the firms in the CDP data are part of the S\&P 500, which does account for a significant amount of total emissions in the US. Some of the firms included in the CDP sample include Wal-Mart (a global retailer), Apache Corporation (an oil and gas company based in 
Texas), PG\&E Corporation (the largest electricity utility company in the US), Cisco Systems (a multinational technology company), and Kraft Foods (one of the largest food and beverage companies in the world). The breakdown of the CDP sample across the ten Global Industry Classification Standard (GICS) sectors mirrors that of the S\&P 500, as shown in Table 1. This shows thatthe fraction of S\&P 500 firms in each GICS sector that report to CDP are close to the fraction of firms in each GICS sector for the S\&P 500 at large.

We compareaverage total assets and the average number of employees between the S\&P 500 firms that reported to CDP in 2013 and the S\&P 500 at large by GICS sector. The CDP sample in Table 1 only includes companies that report complete Scopes 1, 2, and 3. We were able to obtain data from Compustatfor both total assets and the number of employees for 490 companies from the S\&P 500 population. The reason we use total assets is because this is one common measure of firm size and it has been shown that firms with larger total assets are more likely to disclose to CDP (Matsumura et al. 2013, Table 2). We also compare the number of employees because this has also been shown to be related to the likelihood of disclosing to CDP (Dawkins and Fraas 2011). We also observe the same trends here; firms that reported to CDP in 2013 have larger total assets and a higher number of employees, on average, compared to the S\&P 500 population. This means that one should take precaution in extrapolating the results we find here to firms that do not report at all. However, the results we show here are still informative given that CDP and stakeholders rely heavily on data that is disclosed to CDP. 
Table 1:Comparison of number of firms in each Global Industry Classification Standard (GICS) sector, average total assets, and the average number of employees of the S\&P 500 sample of firms that reported to CDP in 2013 and S\&P 500 population in 2013.

\begin{tabular}{|c|c|c|c|c|c|c|c|}
\hline \multirow[t]{2}{*}{ GICS } & \multicolumn{2}{|c|}{ Percent firms reporting } & \multicolumn{3}{|c|}{ Mean Total assets } & \multicolumn{2}{|c|}{$\begin{array}{c}\text { Mean number of } \\
\text { employees }\end{array}$} \\
\hline & CDP & S\&P 500 & & $\overline{\mathrm{CDP}}$ & S\&P 500 & CDP & S\&P 500 \\
\hline & $(\mathrm{N}=245)$ & $(\mathrm{N}=490)$ & \multicolumn{3}{|c|}{ (in millions) } & \multicolumn{2}{|c|}{ (in thousands) } \\
\hline Consumer Discretionary & $15 \%$ & $17 \%$ & $\$$ & 21,394 & 17,572 & 118 & 81 \\
\hline Consumer Staples & $10 \%$ & $8 \%$ & $\$$ & 31,403 & 27,617 & 141 & 120 \\
\hline Energy & $4 \%$ & $9 \%$ & $\$$ & 45,627 & 38,494 & 33 & 17 \\
\hline Financials & $16 \%$ & $17 \%$ & $\$$ & 346,368 & $\$ \quad 214,644$ & 48 & 32 \\
\hline Health Care & $10 \%$ & $10 \%$ & $\$$ & 39,877 & 27,559 & 41 & 32 \\
\hline Industrials & $14 \%$ & $12 \%$ & $\$$ & 23,022 & 31,274 & 69 & 65 \\
\hline Information Technology & $17 \%$ & $14 \%$ & $\$$ & 26,166 & 21,935 & 56 & 46 \\
\hline Materials & $7 \%$ & $6 \%$ & $\$$ & 22,681 & 16,458 & 30 & 24 \\
\hline Telecommunication Services & $2 \%$ & $1 \%$ & $\$$ & 123,287 & 92,147 & 104 & 78 \\
\hline Utilities & $5 \%$ & $6 \%$ & $\$$ & 32,554 & 36,990 & 12 & 13 \\
\hline
\end{tabular}

Notes: Only S\&P 500 companies in 2013 and CDP companies with complete scope reporting are included. We also remove firms that have missing total assets or employee observations.

Second, we will compare the breakdown of emissions derived from the CDP disclosures to the aggregate breakdown in MHW08 and the sector-level breakdown in HWM09. For that comparison to be meaningful, the two samples should be comparable. The firms included in MHW08 and HWM09 represent, in theory, the entire US economy. The firms in the S\&P 500 represent the different industries of the US economy, so the fact that S\&P 500 firms that report to CDP representa similarfraction of firms in each GICS sector to that of the S\&P 500 at large makes the comparison meaningful.

Many of the S\&P 500 firms that report to CDP may operate with global supply chains. The EIOLCA also includes imports and thus captures the emissions associated in outsourcing the 
production of goods and services. Although the EIOLCA may have some limitations that we will discuss in later sections, the EIOLCA approach is one of the most widely used tools for benchmarking the fraction of Scope 3 for the different sectors of the economy (Huang et al. 2009b).

\section{Results and Discussion}

We assess the completeness of Scope 3 reporting to CDP by comparing it to the total emissions reported, relative to external benchmarks estimated by MHW08 and refined by HWM09. These two studies are conducted at the industrylevel. HWM09 refers to an aggregate of many sectors as an industry group. We use the same industry groups as HWM09.

We first compare the proportion of upstream emissions reported to CDP to the average estimated by MHW08. To put that comparison in context, we examine how Scope 3 disclosure has changed over time. We then compare the industry-level breakdown of emissions from the 2013 CDP survey with HWM09.

\subsection{What proportion of their Scope 3 emissions are firms reporting to CDP?}

In the 2013 CDP survey, the average proportion of emissions that fall within Scope 3, across all firms that report emissions from at least one category of upstream Scope 3, is 39\% of the total. (Recall that we eliminate the firms that report no Scope 3 emissions at all; if we included those, the proportion would be even lower.) In other words, if $r_{12}$ is the Scope 1 and 2 emissions

reported to CDP and $r_{3}$ is the reported Scope 3 , then $\frac{r_{3}}{r_{12}+r_{3}}=0.39$, which implies that $r_{3}=$ 
$\frac{0.39}{1-0.39} r_{12}=0.64 r_{12}$. To contrast this with the $74 \%$ obtained by MHW08, assume that the EIOLCA estimate is an appropriate benchmark, so that $\frac{c_{3}}{c_{12}+c_{3}}=0.74$, where $c_{12}$ and $c_{3}$ represent the actual emissions for our CDP sample. It follows that $c_{3}=\frac{0.74}{1-0.74} c_{12}=2.85 c_{12}$. We wish to estimate what proportion of actual Scope 3 emissions firms are reporting, i.e., we want to estimate the ratio $\frac{r_{3}}{c_{3}}$. Given that Scope 1 and 2 are easier to measure and companies have been reporting them for years (cf. Figure 2), it is reasonable to assume that the amount of Scope 1 and 2 reported to CDP is relatively close to the actual emissions, i.e., $r_{12} \approx c_{12}$, in which case $\frac{r_{3}}{c_{3}}=$ $\frac{0.64}{2.85}=0.22$. This would imply that, despite the increasing trend, the 2013 CDP respondents only report $22 \%$ of their actual Scope 3 emissions. The discrepancy can be due to several factors.

First, firms likely under-report their Scope 3 emissions. Their Scope 3 reports to CDP do not need to be complete, and many firms explicitly indicate that they only considered certain categories of emissions, such as business travel (reported by 62\% of US firms in CDP 2013, but only $2 \%$ of total Scope 3 emissions reported).

Second, EIOLCA captures emissions from all upstream tiers of the supply chain. The GHG Protocol encourages firms to engage their tier 1 suppliers to include their upstream suppliers to capture emissions emanating further upstream and to justify any exclusions, but it is unlikely that most firms are able to incorporate much if any information from their tier 2 and further upstream suppliers.

Third, the focus has been on Scope 1 and 2 in the US and global Scope 3. This would mean that the estimates of Scope 3 here are larger than if we were to compare Scope 3 from the US alone. This again would suggest that a significant amount of Scope 3 is not yet being reported. 
Fourth, there could be double counting of Scope 3 emissions in CDP, which EIOLCA avoids. This however would mean that there could potentially be even more underestimation of Scope 3 emissions than we report if double counting in CDP disclosures was removed.

Both the EIOLCA and CDP-based estimates of the breakdown of emissions exhibit considerable uncertainty. The CDP disclosures provide a more accurate representation of what firms see, as this is what they measure and disclose. Both estimates are informative in their own way, and the contrast between the two confirms CDP's (CDP 2014b) comment that firms are not yet fully capturing emissions reductions opportunities in their supply chains.

The preceding analysis relies on the assumption that Scope 1 and 2 CDP disclosures are consistent with the EIOLCA-based estimates in MWH08 and HWM09, i.e., $r_{12} \approx c_{12}$. Even if $r_{12}$ deviates from $c_{12}$ by $50 \%$ in either direction, firms are still only capturing anywhere from $11 \%$ to $34 \%$ of total Scope 3 carbon emissions.

\subsection{How has Scope 3 reporting evolved over time?}

To put the comparison in the previous section in context, we first assess how Scope 3 reporting to CDP has evolved over time, then provide anindustry-level comparison. Starting with the evolution of reporting, Figure 2 already shows that total Scope 3 emissions disclosed are increasing, but this could simply reflect that more firms are disclosing Scope 3 emissions over time, as we see in Figure 1. To better understand the evolution, we investigate the averageindustry-level Scope 3 disclosures from 2005 to 2013. We compute the fraction of emissions that fall within Scope 3 for each industry group, then average that across all industry groups used in HWM09 for each of the CDP reporting years. 
Figure 3 shows that Scope 3 is increasing as a fraction of the total, in a roughly linear fashion, from $15 \%$ in 2005 to $39 \%$ in 2013 . Each observation is a weighted average across all industries, therefore the increase in Scope 3 reflects firms reporting a larger portion of their supply chain emissions rather than an increase in the number of firms reporting. Assuming this linear trend continues, it will take a long time until firms fully capture all emissions related within the supply chain.

Figure 3. Average industry group upstream Scope 3 emissions as \% of total, from CDP disclosures, by CDP reporting year.

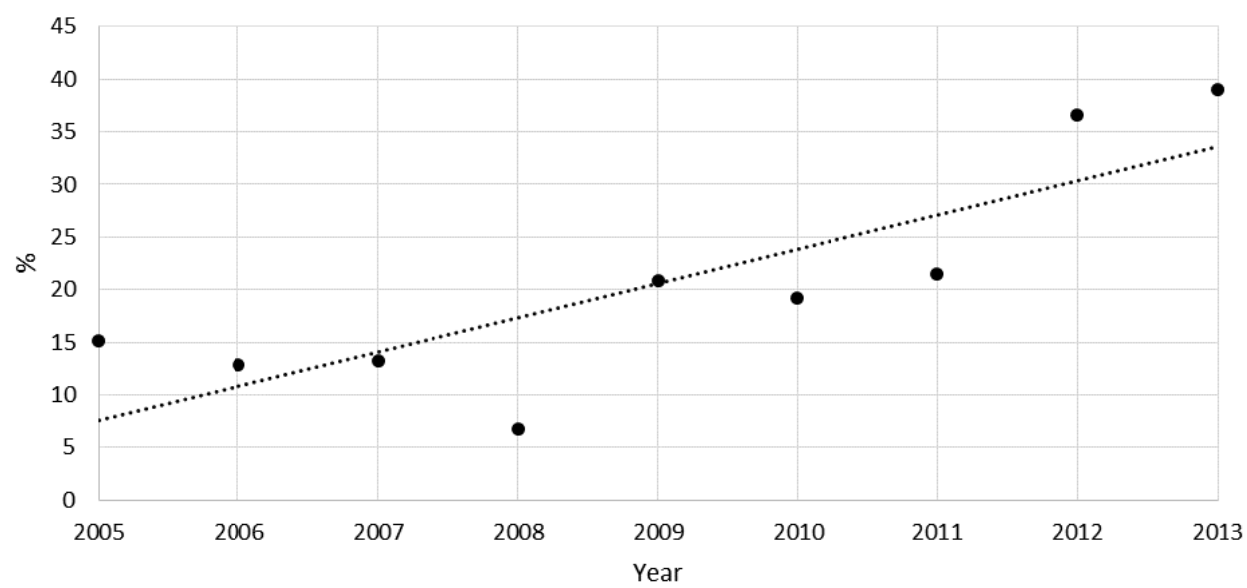

Note: Each observation is a weighted average across all industries, therefore the increase in Scope 3 is attributed to firms capturing a larger portion of their supply chain carbon emissions and not due to the increase in the sample of firms reporting Scope 3 . 


\subsection{How does the discrepancy in Scope 3 reporting vary by industry?}

HWM09 performed a similar analysis as MHW08, but at the level of individual industry groups. They found that the proportion of total emissions (or "total analyzed footprint") that falls within Scope 3 varies widely between industry groups. For example, Scope 3 emissions for the Chemical Products and Drugs industry are estimated to be $73 \%$ of the total, whereas in the Mining industry the estimate is $36 \%$. Given that we found that firms, on average, only report $22 \%$ of the emissions that would be predicted based on EIOLCA, it is natural to ask how that varies across industry groups. Figure 4 shows Scope 3 emissions as a percent of the total carbon emissions for CDP 2013 and HWM09, by industry group (see Figure 1 in HWM09, p.5).

In 11 of the 16 industries in Figure 4, the CDP-based estimate of the proportion of emissions falling in Scope 3 is at least 10 percentage points lower than in HWM09. On average it is 18 percentage points lower; this difference is statistically significant using a Wilcoxon signed rank test $(\mathrm{p}<0.005)$. This means that CDP Scope 3 reports are well below the HWM09 industry estimates.

Industry groups to the right of Figure 4 report significantly lower Scope 3 estimates compared to the breakdown in HWM09. Firms from these industries mostly report Scope 3 from business travel and employee commute. Only 66 firms (or 25\%) reported any positive Scope 3 from purchased goods and services in 2013 , but that source alone accounts for $44 \%$ of total upstream emissions. Similarly, only $25 \%$ report any Scope 3 emissions from transportation and distribution, which appears as one of the top ten sources of emissions for almost every industry group in HWM09. 
Industry groups to the left of Figure 4 report emission breakdowns that are close to or even above the HWM09 estimates. The industry groups to the right of Figure 4 (with the exception of Mining) are more often located downstream, closer to consumers, while industries to the left of Figure 4 are often located further upstream in the supply chain. This observation suggests that the location of an industry within the supply chain may contribute to the difference in Scope 3 estimates between CDP and HWM09. Another factor that may contribute to this difference is the complexity of the upstream supply chain: firms may find it more difficult to collect Scope 3 information if they face many, diversified supply industries.

Figure 4. Completeness of CDP 2013 Scope 3 reporting relative to EIOLCA benchmark.

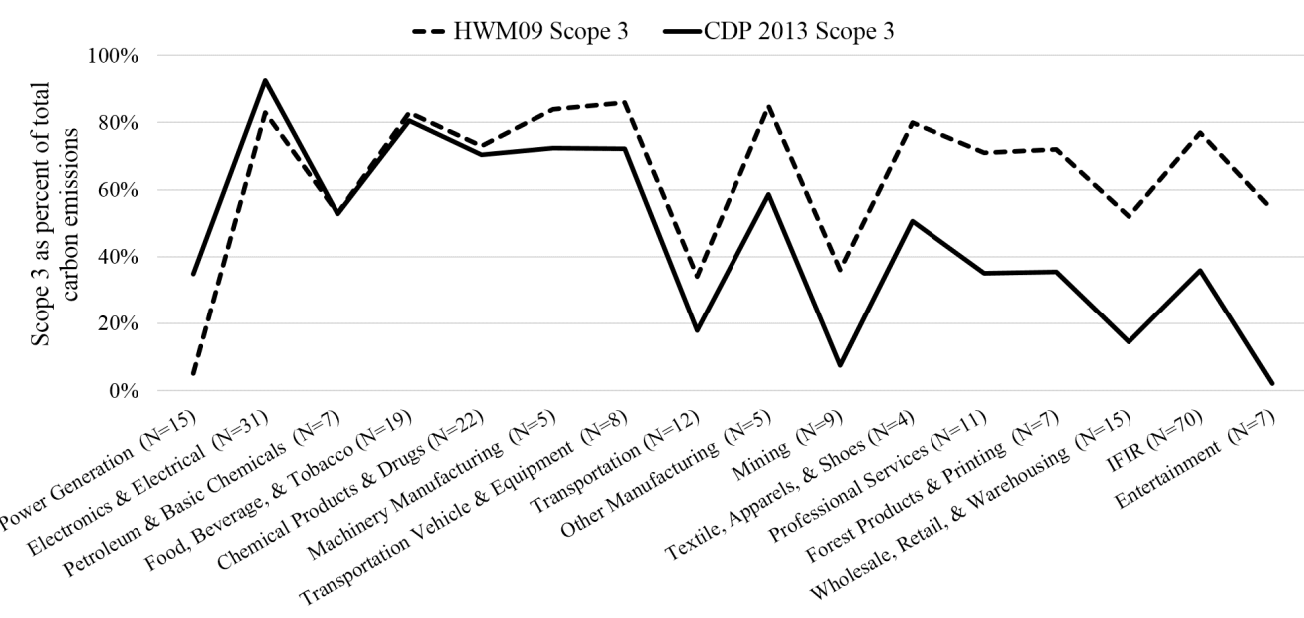

Note: $N$ is the number of firms in each industry group. We include HWM09 industries for which at least four firms reported Scope 3.The correlation between HWM09 Scope 3 and CDP Scope 3 estimates is 0.65. IFIR stands for Information, Financial, Insurance, Real Estate.

In Figure 4 a few industries have very similar breakdowns in CDP as in HWM09. For example, Electronics \& Electrical Equipment ( $\mathrm{N}=31)$, and Food, Beverage, \& Tobacco ( $\mathrm{N}=19)$ 
are within 10 percent of the HWM09 estimates. HWM09 claim that firms may be able to capture a significant portion of their upstream carbon emissions by considering only a few supply sectors. In the Electronics \& Electrical Equipment industry group, Scope 3 accounts for 92\% of total carbon emissions reported to CDP. Two sources account for the majority (90\%) of all Scope 3 emissions: upstream transportation and distribution and purchased goods and services. By focusing on these two categories, the Electronics \& Electrical Equipment industry can indeed capture Scope 3 emissions at a level similar to HWM09.

In addition to the reasons we mentioned earlier that may lead to the discrepancies, we identify two more reasons why the industry estimates may depart from HWM09's estimates. First, HWM09's breakdown, based on EIOLCA with 2002 data, may not be accurate for each

industry (though we do not know any better method). Second, we assign each CDP respondent to an industry, while in practice many firms operate across multiple industries. For example, what is Scope 1 or 2 for a vertically integrated firm would be Scope 3 for another. There could also be mismatching of firms to industries, though that would not cause a systematic bias in the economy-wide breakdown of emissions.

\section{Two Case Studies: Cisco and SC Johnson}

To illustrate how firms can increase the completeness of their reporting in practice, we highlight two firms in different sectors that have significantly expanded their Scope 3 disclosures over time. Cisco and SC Johnson started with Scope 3 reports that were very low in 2010, but, by 2013, they were able to capture a significantly larger fraction of their total supply chain carbon emissions. Moreover, these two companies operate with a large number of suppliers that may 
make Scope 3 difficult to obtain. Cisco's products are almost entirely outsourced to more than 600 suppliers (Cisco 2013). SC Johnson works with large chemical companies such as Dow, Du Pont, and BASF, as well as small specialty chemical companies (SC Johnson 2016). These two firms were still able to capture a significant portion of their Scope 3 carbon emissions despite having a large number of small suppliers or a small number of large suppliers. These two firms are not meant to be representative, but looking at two firms in more detail helps to understand how firms can evolve from reporting little or no Scope 3 to potentially fully disclosing within a few years. An individual firm's emissions breakdown may differ from HWM09's industry-level estimates for many reasons: it may have a different portfolio of products and/or processes than the industry group average, it may be more or less vertically integrated, it may have a different geographical footprint (and hence energy mix), etc. (And the EIOLCA estimates themselves need not be accurate.) Hence, we do not imply that a firm's Scope 3 disclosure is complete if and only if the breakdown matches the industry-level estimate from HWM09.

Cisco has one of the most extensive Scope 3 reports among US firms in 2013. In 2010, Cisco reported upstream Scope 3 from four categories: business travel, capital equipment, energy and fuel (not Scope 1 or 2), and employee commute. They estimated Scope 3 to be $27 \%$ of their total carbon footprint. Despite the fact that Cisco has been reporting to CDP in previous years, and that they have been actively using the GRI guidelines for their sustainability reports, their estimate of the fraction of Scope 3 in 2010 was small, suggesting they did not yet have a lot of experience measuring supply chain carbon emissions. For example, Cisco mentions in their sustainability report that their earlier reports of Scope 1, 2, and 3 data vary compared to earlier reported values because of changes in methodology (Cisco 2010, p. 31). However, they continued to improve the way they measure their carbon emissions, including those in the supply 
chain, as mentioned in their reports: "Cisco is now focusing resources to better characterize measure and report indirect emissions categories including emissions from Cisco's supply chain and product use" (Cisco 2010, p. 31). In 2011, they added Scope 3 from upstream transportation and distribution, capturing a larger portion of supply chain carbon emissions. The increasing alignment of the GRI and the CDP reporting framework is also reflected in Cisco's 2011 sustainability reports. For example, Cisco referred to GRI EN3 and GRI EN16 as "operations Scope 1 and 2" and identified GRI EN17 as "Scope 3" and GRI EN29 as "Scope 3: Business Air travel" (Cisco 2011, p. F10, F14). During that time, the GRI was not yet updated to the G4 to reflect how the same data can be used in either reporting framework, but Cisco had already done so. In 2013, Cisco reported upstream Scope 3 emissions to be $79 \%$ of their total disclosed emissions. This is very close to HWM09's estimate of approximately $80 \%$ for the Electronics \& Electrical Equipment industry group.

Because of the diverse number of firms that supply Cisco (Cisco 2015), they would need to reach out to many suppliers to capture a large portion of their upstream Scope 3. According to Cisco's qualitative CDP response, this is exactly what they did, using a combination of surveys and purchase information from their suppliers.

SC Johnson followed a similar evolution. SC Johnson mention that they prepared their data for their 2009 and 2010 corporate social reports following the 2002 GRI Sustainability Reporting Guidelines (SC Johnson 2009, p. 46; SC Johnson 2010 p. 17), but they do not yet mention the GHG Protocol. In 2009, SC Johnson acknowledged the importance of measuring supply chain carbon emissions and participated in developing reporting standards for Scope 3: "SC Johnson is working with the World Resources Institute and the World Business Council for Sustainable Development to develop global standards for measuring and reporting GHG emissions within the 
supply chain." (SC Johnson 2009, p. 27). SC Johnson volunteered to implement early stages of the standardin 2010 (SC Johnson 2010, p. 13). SC Johnson did not yet disclose any Scope 3 emissions in 2010, but in 2011, they estimated Scope 3 to be $92 \%$ of their total carbon emissions. They were able to capture a large portion of their Scope 3 carbon emissions using surveys. SC Johnson sent out questionnaires asking for their suppliers' Scope 1 and 2 emissions to determine their own Scope 3 emissions from purchased goods and services. Based on their qualitative response to the CDP 2012 survey, SC Johnson contacted 92 suppliers and contract manufacturers, of which 66 provided data. In 2013, SC Johnson's reported upstream Scope 3 accounted for more than $90 \%$ of the company's total.

These examples show how supplier surveys can be used to more fully capture all emissions within the supply chain. Beginning with suppliers furthest upstream, Scope 3 emissions can be added along the supply chain through "backpacking" (Schmidt 2009, p. 3). The most upstream firm reports their Scope 1 and 2 to their first tier of buyers, for whom those emissions are Scope 3; those buyers add those Scope 3 emissions to their own Scope 1 and 2, into a "backpack" of emissions that they pass on to their buyers, continuing until all emissions have been backpacked across the supply chain (Schmidt 2009). The likelihood of voluntary disclosure increases as the number of industry peers disclosing increases (Jira and Toffel 2013; Matsumura et al. 2013), so if protocol organizations can highlight firms with more comprehensive Scope 3 reports, other firms may be more likely to increase their own Scope 3 reporting. 


\section{Conclusions and Future Directions}

We provided a summary of the evolution and current state of carbon emissions reporting in supply chains by many of the largest firms in the US. We used data from the CDP surveys to estimate the fraction of emissions falling within Scope 3, which others have claimed often substantially exceeds companies' emissions from directly owned equipment and energy purchases (Hoffmann and Busch 2008; Huang 2009a; Matthews et al. 2008). We showed that US firms disclosing positive Scope 3 emissions to CDP in 2013 reported about $22 \%$ of the Scope 3 emissions that would be expected using a benchmark from MHW08. We found that Scope 3 accounted for an increasing proportion of total emissions reported, growing from $15 \%$ in 2005 to $39 \%$ in 2013. We also found significant variation in Scope 3 estimates by sector, suggesting that industry-specific characteristics of supply chains may affect the completeness of Scope 3 reporting, a topic for further investigation.A key managerial insight from out paper is that firms that have a large number of suppliers can still successfully capture a significant portion of their supply chain carbon emissions as illustrated by the two case studies. We highlightedhow twofirms were able to capture a larger portion of their upstream supply chain emissions using supplier surveys to broaden their reach.

Although the MHW08 benchmark is not an accurate "target", it provides evidence that the majority of a firm's carbon emissions are likely to come from their upstream supply chain. Taken together, our findings help to provide benchmarks for a firm's Scope 3 emissions reports and to assess the extent to which firms are capturing the risks and opportunities associated with carbon emissions within their supply chain. We find that more firms are beginning to account for a larger portion of their supply chain carbon emissions, but there are still opportunities for firms to 
measure and disclose a larger portion of their upstream Scope 3. Given the observations by Seuring and Müller (2008), Seuring and Gold (2013), and others, that increased collaboration and performance measurement across entire supply chains is needed to make them more sustainable, our findings suggest that firms still have a long way to go in this regard, especially as carbon may be one of the better-measured sustainability metrics.

According to HWM09, firms can focus on a handful of suppliers and still be able to capture a significant portion of emissions from the supply chain. HWM09 show in their supplemental material that the top-10 supply sectors that account for the total carbon emissions varies by industry. We also find that the fraction of Scope 3 reported by firms belonging to industries that are often considered more downstream, closer to the consumer, have significantly lower fraction of Scope 3 estimated compared to that of HWM09. Future studies can explore if the top-10 supply sectors influence the completeness of Scope 3 reporting. It would be worthwhile to explore if industries that have a more diversified number of suppliers are less likely to capture a significant portion of their Scope 3 emissions.

Firms may also benefit from the alignment of carbon emissions standards across different reporting frameworks. There are currently many environmental reporting platforms such as the CDP and the GRI, and these platforms have become increasing compatible, lessening the burden of carbon emissions disclosure. For example, the recent changes in the GRI guidelines reflect the increasing shift in attention to supply chain carbon emissions (GRI 2013). An alternative approach to assessing the quality and completeness of carbon emissions reporting is to compare how company reports have evolved over time between the CDP and the GRI frameworks. Future areas of research can explore if firms are more likely to report environmental impact or carbon emissions if they can use the same data in multiple reporting platforms. 
Our approach can also be extended to other countries and regions. For example, our approach of benchmarking Scope 3 emissions can be extended to other countries or to multiregional EIOLCA models (Wiedmann et al. 2007). Our benchmark only includes upstream carbon emissions, but emissions related to consumers are also of great importance. Some works have begun exploring the importance of carbon footprinting at the national level. For example, Sanches-Pereira et al. (2016) estimate the carbon footprint related to residential energy consumption. Our approach of using firm-level data can be used in conjunction with studies that estimate consumer-related carbon emissions. Our focus in this study has been on upstream Scope 3 carbon emissions, but companies also report Scope 3 related to the use of goods and services (downstream, carbon emissions). This data can be used with other engineering-economic estimates of carbon emissions related to the use of goods and services. We believe this intersection of EIOLCA models and firm-reported data is a rich area for research.

\section{Acknowledgements}

We are grateful to CDP for providing access to their dataset, in particular to Patrick Crawford, Maia Kutner, and Emma Whelan for helpful discussions and assistance with the data and to Kristian Hardiman for helpful comments. We also thank Anny Huang for providing numerical values from Figure 1 of HWM09, and to Paulina Jaramillo for helpful suggestions. We acknowledge research funding from the UCLA Anderson Center for Global Management and the UCLA Anderson Easton Technology Leadership Program. Christian Blanco was partially funded by the National Science Foundation Graduate Research Fellowship (NSF GRFP) Grant No. 2011131079. 


\section{References}

Brown., L.H., Buettner, P.G., Canyon, D.V., Mac Crawford, J., \& Judd, J. (2012). Estimating thelife cycle greenhouse gas emissions of Australian ambulance services. Journal ofCleaner Production, 37, 135-141.

Basacik, L., Kutner, M., Buck, B., Dreyfus, R., Espinach, L., Hagen, S., Kriege, K. 2015. Linking GRI and CDP: How are GRI's G4 Guidelines and CDP's 2015 Climate Change Questions Aligned? https://www.globalreporting.org/resourcelibrary/GRI-G4-CDP-2015Climate-Change-Linkage-Document.pdf. Last accessed February 2016.

Boström, M., Jönsson, A. M., Lockie, S., Mol, A. P., Oosterveer, P. 2015. Sustainable and responsible supply chain governance: challenges and opportunities. Journal of Cleaner Production 107, 1-7.

Busch, T. 2010. Corporate carbon performance indicators revisited. Journal of Industrial Ecology 14(3), 374-377.

Busch, T. 2011. Which emissions do we need to account for in corporate carbon performance?

Response to Murray and colleagues. Journal of Industrial Ecology 15(1), 160-163.

CDP. 2009. Carbon Disclosure Project 2009 S\&P 500 Report.

https://www.cdp.net/CDPResults/CDP\%202009\%20SandP500\%20with\%20Industry\%20

Snapshots.pdf. Last accessed February 2016.

CDP. 2012. CDP S\&P 500 Climate Change Report 2012.https://www.cdp.net/CDPResults/CDPSP500-2012.pdf. Last accessed February 2016.

CDP. 2013a. Investor CDP 2013 information request. 
https://www.cdp.net/CDP\%20Questionaire\%20Documents/Investor-CDP-2013-

Information-Request.pdf. Last accessed February 2016.

CDP. 2014a. CDP Report 2013, S\&P 500.www.cdp.net/CDPResults/CDP-SP500-climate-report2013.pdf.Lastaccessed August 2014.

CDP. 2014b. CDP Report 2013, Global 500.www.cdp.net/cdpresults/cdp-global-500-climatechange-report-2013.pdf. Last accessed August 2014.

CDP. 2016. CDP Academic data package. https://www.cdp.net/en-US/Results/Pages/academicdata.aspx. Last accessed February 2016.

Cisco. 2010. Cisco 2010 Corporate Social Responsibility Report. http://www.cisco.com/assets/csr/pdf/CSR_Report 2010.pdf. Last accessed February, 2016.

Cisco. 2011. Cisco 2011 Corporate Social Responsibility Report. http://www.cisco.com/assets/csr/pdf/CSR_Report_2011.pdf. Last accessed February, 2016.

Cisco. 2013. Cisco 2013 Corporate Social Responsibility Report. http://www.cisco.com/assets/csr/pdf/CSR_Report 2013.pdf. Last accessed February, 2016.

Cisco. 2015. Focus Area: Supply Chain.http://csr.cisco.com/pages/supply-chain. Last accessed August 6, 2015.

Dawkins, C., \&Fraas, J. W. (2011). Coming clean: The impact of environmental performance and visibility on corporate climate change disclosure. Journal of Business Ethics, 100(2), 303-322. 
Downie, J., Stubbs, W. 2013. Evaluation of Australian companies' scope 3 greenhouse gas emissions assessments. Journal of Cleaner Production, 56, 156-163.

Dragomir, V.D. 2012. The disclosure of industrial greenhouse gas emissions: a critical assessment of corporate sustainability reports. Journal of Cleaner Production 29, 222237.

GRI. 2013. Streamlining sustainability: Increased focus on supply chain management and disclosure. https://www.globalreporting.org/information/news-and-presscenter/Pages/Streamlining-sustainability-Increased-focus-on-supply-chain-managementand-disclosure.aspx. Last accessed February 2016.

GRI. 2015. G4 Sustainability Reporting Guidelines. https://www.globalreporting.org/resourcelibrary/GRIG4-Part1-Reporting-Principles-and-

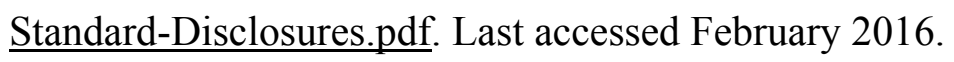

Güereca, L. P., Torres, N., \&Noyola, A. (2013). Carbon footprint as a basis for a cleaner research institute in Mexico. Journal of Cleaner Production, 47, 396-403.

Hoffmann, V. H., Busch, T. 2008. Corporate carbon performance indicators.Journal of Industrial Ecology 12(4), 505-520.

Huang, Y. A., Weber, C. L., Matthews, H. S. 2009a. Categorization of scope 3 emissions for streamlined enterprise carbon footprinting. Environmental Science \&Technology 43(22), 8509-8515.

Huang, Y. A., Lenzen, M., Weber, C. L., Murray, J., Matthews, H. S. 2009b. The role of inputoutput analysis for the screening of corporate carbon footprints. Economic Systems Research 21(3), 217-242. 
Jira, C., Toffel, M. W. 2013. Engaging supply chains in climate change.Manufacturing \& Service Operations Management 15(4), 559-577.

Kolk, A., Levy, D., Pinkse, J. 2008. Corporate responses in an emerging climate regime: the institutionalization and commensuration of carbon disclosure. European Accounting Review 17(4), 719-745.

Marland, G., Buchholz, T., Kowalczyk, T. 2013. Accounting for carbon dioxide emissions. Journal of Industrial Ecology 3, 340-342.

Matisoff, D. C., Noonan, D. S., O'Brien, J. J. 2013. Convergence in environmental reporting: assessing the Carbon Disclosure Project. Business Strategy and the Environment 22(5), 285-305.

Matsumura, E. M., Prakash, R., Vera-Muñoz, S. C. 2013. Firm-value effects of carbon emissions and carbon disclosures. The Accounting Review 89(2), 695-724.

Matthews, H. S., Hendrickson, C. T., Weber, C. L. 2008. The importance of carbon footprint estimation boundaries. Environmental Science \&Technology 42(16), 5839-5842.

Melville, N. P., Whisnant, R. 2014. Energy and Carbon Management Systems. Journal of Industrial Ecology 18(6), 920-930.

Murray, J., Wiedmann, T., Dey, C. 2011. Comment on "corporate carbon performance indicators revisited". Journal of Industrial Ecology 15(1), 158-160.

Onat, N. C., Kucukvar, M., \&Tatari, O. (2014). Scope-based carbon footprint analysis of US residential and commercial buildings: An input-output hybrid life cycle assessment approach. Building and Environment, 72, 53-62. 
Ozawa-Meida, L., Brockway, P., Letten, K., Davies, J., Fleming, P. 2013. Measuring carbon performance in a UK University through a consumption-based carbon footprint: De Montfort University case study. Journal of Cleaner Production 56, 185-198.

Plambeck, E. L. 2012. Reducing greenhouse gas emissions through operations and supply chain management. Energy Economics, 34, S64-S74.

Reid, E. M., Toffel, M.W. 2009. Responding to Public and Private Politics: Corporate Disclosure of Climate Change Strategies. Strategic Management Journal 30, 1157-1178.

Sanches-Pereira, A., Tudeschini, L. G., \& Coelho, S. T. (2016). Evolution of the Brazilian residential carbon footprint based on direct energy consumption. Renewable and Sustainable Energy Reviews, 54, 184-201.

Schmidt, M. 2009. Carbon accounting and carbon footprint-more than just diced results? International Journal of Climate Change Strategies and Management 1(1), 1930.

SC Johnson. 2009. SC Johnson Public Report 2009. https://www.scjohnson.com/Libraries/Download_Documents/2009_SC_Johnson_Public Report.sflb.ashx. Last accessed February 2016.

SC Johnson. 2010. SC Johnson Public Report 2010. https://www.scjohnson.com/Libraries/Download_Documents/SCJohnson-PublicReportPlanet.sflb.ashx. Last accessed February 2016.

SC Johnson. 2016. SC Johnson is transforming its supply chain to create products that are better for the environment. http://www.greenchemistryandcommerce.org/downloads/SCJ_final.pdf. Last accessed February 2016. 
Searcy, D. L., \&Mentzer, J. T. (2003). A framework for conducting and evaluating research. Journal of Accounting Literature, 22, 130.

Seuring, S., Gold, S. 2013. Sustainability management beyond corporate boundaries: from stakeholders to performance. Journal of Cleaner Production 56, 1-6.

Seuring, S., Müller, M. 2008. From a literature review to a conceptual framework for sustainable supply chain management. Journal of Cleaner Production 16, 1699-1710.

Stechemesser, K., Guenther, E. 2012. Carbon accounting: a systematic literature review. Journal of Cleaner Production 36, 17-38.

Sullivan, R., Gouldson, A. 2012. Does voluntary carbon reporting meet investors' needs? Journal of Cleaner Production 36, 60-67.

Talbot, D., Boiral, O. 2013. Can we trust corporates GHG inventories? An investigation among Canada's large final emitters. Energy Policy 63, 1075-1085.

Tidy, M., Wang, X., \& Hall, M. (2016). The role of Supplier Relationship Management in reducing Greenhouse Gas emissions from food supply chains: supplier engagement in the UK supermarket sector. Journal of Cleaner Production, 112, 3294-3305.

Vachon, S., Klassen, R. D. 2008. Environmental management and manufacturing performance: the role of collaboration in the supply chain.International Journal of Production Economics 111(2), 299-315.

Wiedmann, T., Lenzen, M., Turner, K., \& Barrett, J. (2007). Examining the global environmental impact of regional consumption activities-Part 2: Review of inputoutput models for the assessment of environmental impacts embodied in trade. Ecological economics, 61(1), 15-26. 
Wilson, M. C. (2013). A Critical Review of Environmental Sustainability Reporting in the Consumer Goods Industry: Greenwashing or Good Business?. Journal of Management and Sustainability, 3(4), 1.

WRI/WBCSD. 2004. “The Greenhouse Gas Protocol: ACorporate Accounting and Reporting Standard (revised edition).” Washington, DC: World Resources Institute and World Business Council for Sustainable Development (2004). Last accessed February 2015, http://ghgprotocol.org/files/ghgp/public/ghg-protocol-revised.pdf.

WRI/WBCSD. 2015. "Corporate Value Chain (Scope 3) Accounting and Reporting Standard. Supplement to the GHG Protocol Corporate Accounting and Reporting Standard". www.ghgprotocol.org/files/ghgp/public/Corporate-Value-Chain-Accounting-ReporingStandard 041613.pdf. Last accessed October 28, 2015.

Yang, J., \& Chen, B. (2014). Carbon footprint estimation of Chinese economic sectors based on a three-tier model. Renewable and Sustainable Energy Reviews, 29, 499-507. 